\title{
Alanine Aminopeptidase Measurement
}

National Cancer Institute

\section{Source}

National Cancer Institute. Alanine Aminopeptidase Measurement. NCI Thesaurus. Code C100430.

The determination of the amount of alanine aminopeptidase present in a sample. 